\title{
Orbital Period Variation in the Chromospherically Active Binary FF UMa (2RE J0933+624)
}

\author{
M.C. Gálvez ${ }^{1,2}$, D. Montes $^{1}$, M.J. Fernández-Figueroa ${ }^{1}$, \\ E. De Castro ${ }^{1}$ and M. Cornide ${ }^{1}$ \\ ${ }^{1}$ Departmento de Astronomía, Facultad de Físicas, Universidad Complutense de Madrid, \\ Madrid, Spain \\ email: mcz@astrax.fis.ucm.es \\ ${ }^{2}$ Department of Astronomy, University of Florida, Bryant Space Science Center, \\ Gainesville, Florida, USA \\ email:mcz@astro.ufl.edu
}

\begin{abstract}
We present here a detailed study of FF UMa (2RE J0933+624), a recently discovered, $\mathrm{X}$-ray/EUV selected, active binary system with strong $\mathrm{H} \alpha$ emission. By using high-resolution echelle spectroscopic observations taken during five observing runs from 1998 to 2004, we derived precise radial velocities that allowed us to determine the orbital solution of the system at different epochs. Analyzing these orbital solutions, together with a previous one determined in 1993 by other authors, we have found a change in the heliocentric Julian date on conjunction $\left(T_{\text {conj }}\right)$ that can be explained by a change with time in the orbital period of the system. The relative amplitude of the orbital period variation derived from these data was $d P / P \approx 5 \times 10^{-4}$, which is larger than the variations found in other similar chromospherically active binaries like AR Lac and HR 1099. This orbital period variation can be related (Applegate 1992; Lanza 1998, 2006) with the modulation of the gravitational quadrupole-moment of its magnetically active secondary star produced by angular momentum exchanges within its convective envelope. In addition, using these observations, we have determined the stellar parameters of the components and we have carried out a study of the chromospheric activity using all the optical indicators from $\mathrm{Ca}$ II $\mathrm{H} \& \mathrm{~K}$ to $\mathrm{Ca}$ II IRT lines.
\end{abstract}

Keywords. stars: binaries: spectroscopic, stars: activity, stars: atmospheres, stars: chromospheres, stars: late-type, stars: fundamental parameters

\section{Observations}

Spectroscopic observations of this binary system were obtained during five observing runs from 1998 to 2004:

- 12 to 21 January 1998 using the 2.1-m Otto Struve Telescope at McDonald Observatory (Texas, USA) with the Sandiford Cassegrain Echelle Spectrometer. During this observing run, a $1200 \times 400$ pixel CCD detector was used. The spectrograph setup was chosen to cover the $\mathrm{H} \alpha(6563 \AA)$ and Ca II IRT $(8498,8542,8662 \AA)$ lines. The wavelength range covers from 6400 to $8800 \AA$ in 31 orders. The reciprocal dispersion ranges from 0.06 to $0.08 \AA$ /pixel and the spectral resolution, determined as the FWHM of the arc comparison lines, ranges from 0.13 to $0.20 \AA$. On one of the nights, we changed the spectrograph setup to include the He I $\mathrm{D}_{3}(5876 \AA)$ and Na I $\mathrm{D}_{1}, \mathrm{D}_{2}(5889.9,5895.9 \AA)$, with wavelength coverage of $5600-7000 \AA$.

- 22 to 24 January 2000 using the 9.2-m Hobby-Eberly Telescope (HET) at McDonald Observatory, with the medium-resolution spectrograph UFOE (Upgraded Fiber Optic Echelle). A $1200 \times 400$ pixel CCD detector was used. The wavelength range covers from 

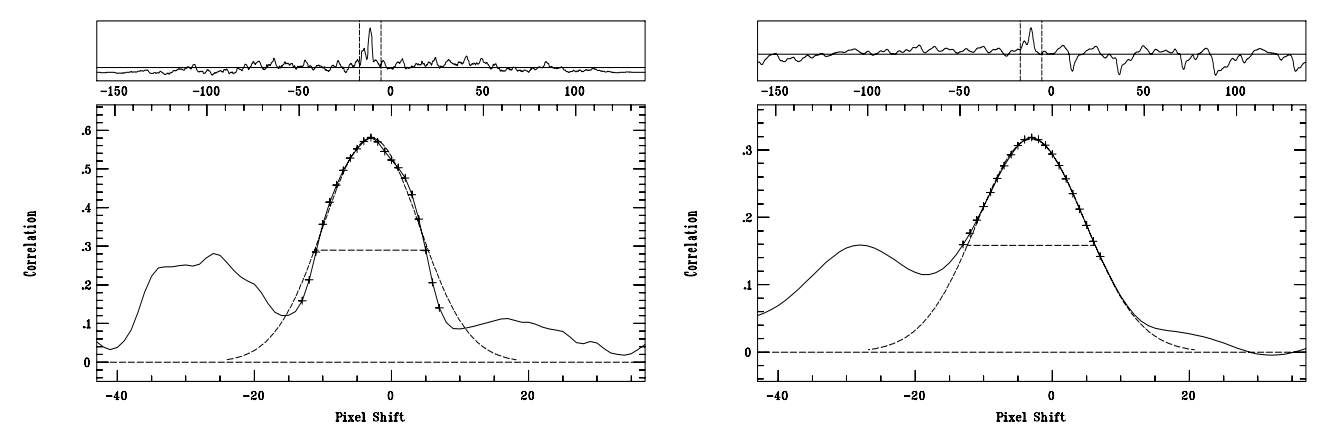

Figure 1. Cross-correlation functions (CCFs) of the both components of the binary system, fitted with Gaussians. ( $a$ ) left panel, original CCF, $(b)$ right panel, corrected CCF.

4400 to $9150 \AA$ in 26 orders. The reciprocal dispersion ranges from 0.06 to $0.17 \AA /$ pixel and the spectral resolution (FWHM) from 0.14 to $0.42 \AA$.

- 22 to 26 April 2002 and 29 March to 7 April 2004, using the 2.2-m telescope at the German Spanish Astronomical Observatory (CAHA) (Almeria, Spain). The Fibre Optics Cassegrain Echelle Spectrograph (FOCES) was used with a $2048^{2} 24 \mu$ SITE\#1d CCD detector. The wavelength range covers from 3450 to $10700 \AA$ in 112 orders. The reciprocal dispersion ranges from 0.04 to $0.13 \AA$ /pixel and the spectral resolution (FWHM) ranges from 0.08 to $0.35 \AA$.

- 2 to 6 April 2004, using the 2.56-m Nordic Optical Telescope (NOT) located at the Observatory Roque de Los Muchachos (La Palma, Spain). The Soviet Finnish High Resolution Echelle Spectrograph (SOFIN) was used with an echelle grating (79 grooves $/ \mathrm{mm}$ ), ASTROMED-3200 camera and a 20522 pixel 2K3EB PISKUNOV1 CCD detector. The wavelength range covers from 3545 to $10120 \AA$ in 42 orders. The reciprocal dispersion ranges from 0.033 to $0.11 \AA /$ pixel and the spectral resolution (FWHM) from 0.14 to 0.32 $\AA$.

\section{Radial velocities}

Heliocentric radial velocities of both components have been determined by using the cross-correlation technique. The spectra of the program stars were cross-correlated order by order, using the routine FXCOR in IRAF, against spectra of radial velocity standards of similar spectral types. The velocity is derived from the position of the cross-correlation peak (Figures 1a, 1b). As the system is SB2 we can see two peaks in the CCF coming from the two components and we can fit each peak separately. When the components are too close, we used deblend fits. But as it can be seen in the Figure 1a the irregulars profiles (double peaks and asymmetries) can produce errors in radial velocity measures. To correct this error, we recalculated the radial velocities using the cross-correlation technique against spectra of the same radial velocity standard star but rotational broadened to the rotational velocity of the components of FF UMa $\left(v \sin i \approx 30 \mathrm{~km} \mathrm{~s}^{-1}\right)$. In this way the profiles of the CCF become regular; see Figure $1 \mathrm{~b}$.

\section{Orbital Period Variation}

With 39 radial determined by us (from 1998 to 2004) and 9 given by Jeffries et al. (1995), we tried to compute the orbital solution but we found some complications. When we fit the data from each observing run separately we obtained a good orbital solution, 


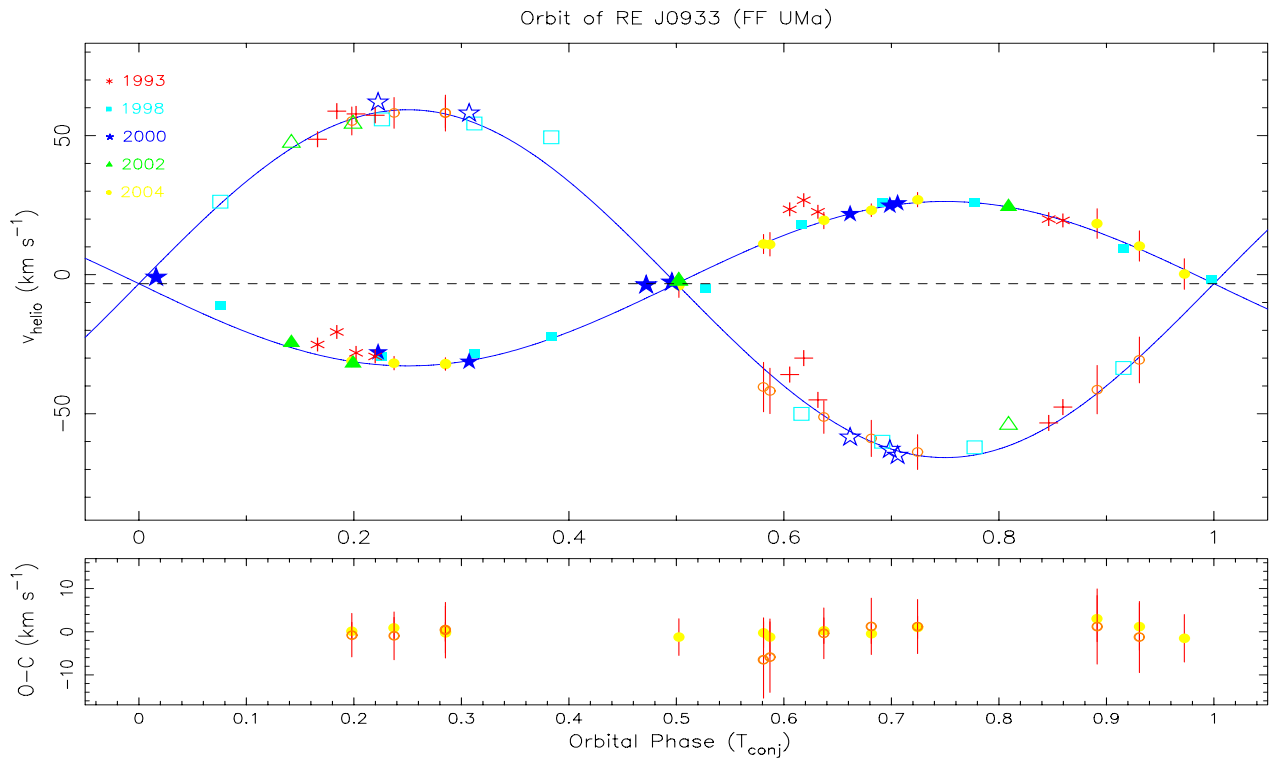

Figure 2. Radial velocities of all observing runs. We plotted the orbital solution (continuum line) of the FOCES04 observing run and we have superimposed the data from the rest runs shifted in phase according to the determined $O-C$ in $T_{\text {conj }}$; see text for explanation. Different symbols and colours correspond to different epochs: 1993 (Jeffries 1995) and 1998-2004 (our observing runs).

Table 1. Orbital solution

\begin{tabular}{llll}
\hline Element & Value & Uncertainty & Units \\
\hline$P_{\text {orb }}$ & 3.274 & 0.054 & days \\
$T_{\text {conj }}$ & 53090.84 & 0.18 & HJD $(2400000+)$ \\
$\omega$ & 0.00 & 0.00 & degrees \\
$e$ & 0.00 & 0.00 & (adopted) \\
$K_{1}$ & 29.55 & 0.95 & $\mathrm{~km} \mathrm{~s}^{-1}$ \\
$K_{2}$ & 62.52 & 3.60 & $\mathrm{~km} \mathrm{~s}^{-1}$ \\
$\gamma$ & -3.23 & 0.77 & $\mathrm{~km} \mathrm{~s}^{-1}$ \\
$q=M_{1} / M_{2}$ & 2.12 & 0.10 & \\
$a_{1} \sin i$ & 1.330 & 0.048 & $10^{6} \mathrm{~km}$ \\
$a_{2} \sin i$ & 2.81 & 0.17 & $10^{6} \mathrm{~km}$ \\
$a \sin i$ & 4.14 & 0.18 & $10^{6} \mathrm{~km}$ \\
$"$ & 0.028 & & $\mathrm{AU}$ \\
& & & $\mathrm{R}_{\odot}$ \\
$M_{1} \sin ^{3} i$ & 0.180 & 0.024 & $M_{\odot}$ \\
$M_{2} \sin ^{3} i$ & 0.085 & 0.012 & $M_{\odot}$ \\
$\mathrm{f}(M)$ & 0.00875 & 0.00099 & $M_{\odot}$ \\
\hline
\end{tabular}

but when we tried to fit the total orbital solution (using all the radial velocities from the different epochs) we were not able to find a good orbital solution. Analyzing in detail these results we discovered that orbital parameters obtained at different epochs were very similar, but there was a shift in the heliocentric Julian date of conjunction $\left(T_{\text {conj }}\right)$. See Figures 2 and 3 . 


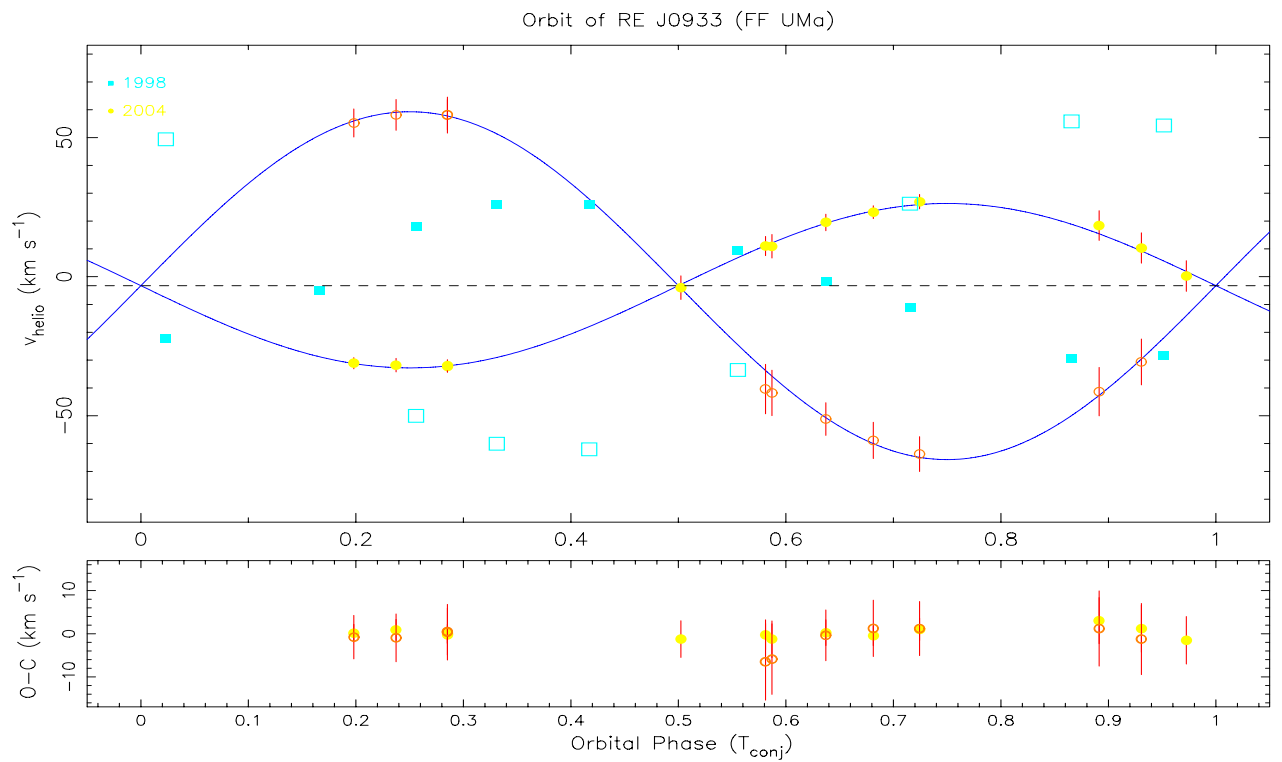

Figure 3. Note the orbital phase shift between the orbital solution with the data of the FOCES04 observing run (continuous line) and McDonald 1998 (blue squares)

Taken this into account, we computed the orbital solution with the FOCES04 run and shifted in phase the rest of the observing runs (see Section 4). We have analyzed the different hypothesis that can explain this behaviour:

- The existence of another star as a distant third component of the system could introduce changes in the main orbit of the observed system. Variations in in the center of mass radial velocity $(\gamma)$ could indicate the presence of a third component (Cumming 2004). However, the amplitude of the variations in $\gamma$ we have found in our data is only $3 \mathrm{~km} \mathrm{~s}^{-1}$.

- The presence of photospheric starspots in the active components of this binary could produce asymmetries in the absorption line profiles and the corresponding CCF. This could affect the radial velocity data and in this way transmit errors to the orbital solutions. However, we have found (see Section 2) that this is not the case.

- Another explanation associated to the magnetic activity could be an orbital period modulation due to the variation of activity with time. It can be explained as a consequence of cyclic variations of the quadrupole-moment of both components of the system along the magnetic activity cycle. In their study of the RS CVn system HR 1099, Frasca \& Lanza (2005) based their findings on the hypothesis presented by Matese \& Whirtmere (1983) and elaborated upon later by Applegate (1992) and Lanza et al. (1998), in which temporal variations of some RS CVn systems could be due to changes in the quadrupole moment of the primary component along the activity cycle, which results from the exchange between kinematics and magnetic energy driven by the stellar hydromagnetic dynamo. The results obtained until this study were variations of $d P / P \approx 10^{-6}-10^{-5}$ over an interval of 7 to 109 years. In FF UMa we have found a variation of $d P / P \approx 10^{-4}$ over 11 years; this is one order of magnitude higher, but it could be explained by the higher efficiency of the dynamo mechanism in the components of the system.

In Table 2 we present the orbital period variation deduced from the orbital solution of the different observing runs. In the first column we give the year of the observing run, in the second, $T_{\text {conj }}$, in the third, the $O-C$ (Observed - Calculated $=$ difference between 


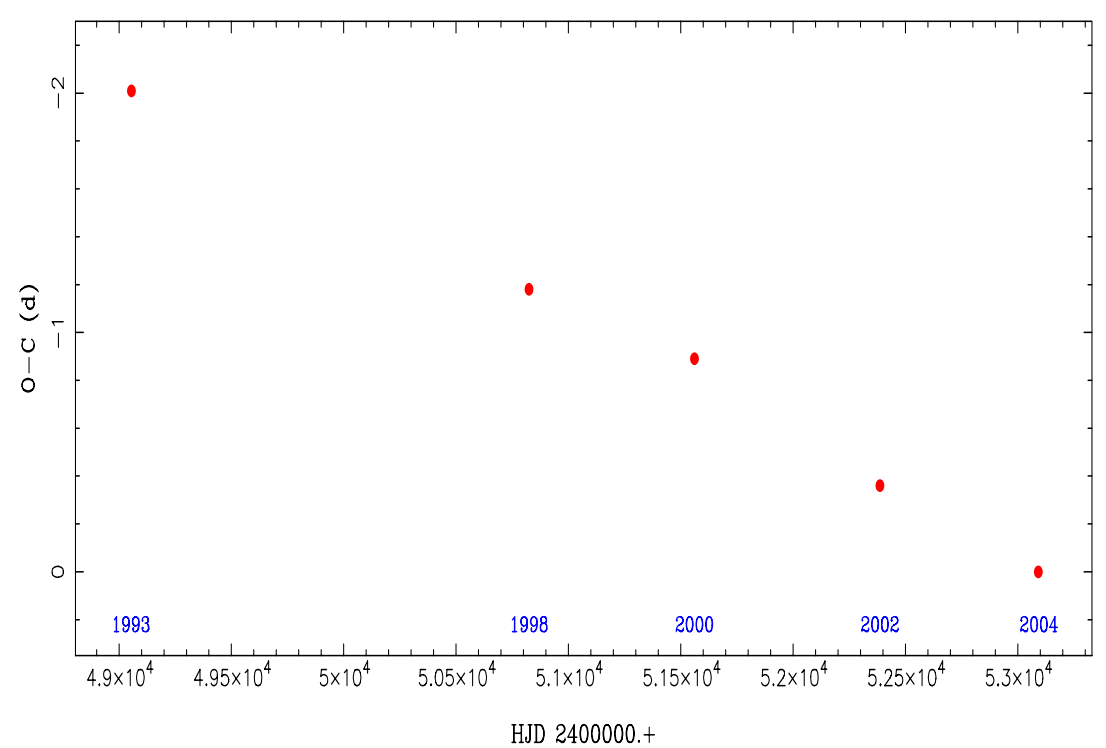

Figure 4. Period variation representation. The difference (O-C) is plotted against Heliocentric Julian date HJD for every observing run.

Table 2. Variations of Orbital Period $(\mathrm{P})$

\begin{tabular}{cccc}
\hline Year & $\begin{array}{c}T_{\text {conj }} \\
\text { HJD } \\
(2400000+)\end{array}$ & $\begin{array}{c}\text { O-C } \\
\text { day }\end{array}$ & $d P / P$ \\
\hline 1993 & 49055.8789 & -2.0089 & $4.979 \times 10-4$ \\
1998 & 50824.4023 & -1.1755 & $5.187 \times 10-4$ \\
2000 & 51561.2227 & -0.9005 & $5.887 \times 10-4$ \\
2002 & 52386.6758 & -0.3615 & $5.134 \times 10-4$ \\
2004 & 53090.8398 & & \\
\hline
\end{tabular}

the observed $T_{\text {conj }}$ and calculated $T_{\text {conj }}$, from the FOCES04 run that has been taken as reference), and in the last column the relative orbital period variation, $d P / P$. Figure 4 . represents the variation of the $O-C\left(T_{\text {conj }},\right)$ for every run with time. The amplitude of the variation is 2 days and it look like a linear tendency. We would need observations over a longer time to test if the tendency remains linear or becomes sinusoidal, as would be expected if there is a cyclical motion.

Although recent studies, such as that by Lanza (2006), are not completely in agreement with the Applegate model, we found that the quantitative explanation of the phenomena is the most likely for the FF UMa system.

\section{Orbital Solution}

We have computed the orbital solution of this system using radial velocities data from the FOCES 2004 observing run. The radial velocity data are plotted in Figure 2. Solid symbols represent the primary and open symbols represent the secondary; each observing run is represented by a different symbol. The curve represents a minimum $\chi^{2}$ fit orbit solution. The orbital solution and relevant derived quantities are given in Table 1 and Figure 2. In this table we give $T_{\text {conj }}$ as the heliocentric Julian date of conjunction with 

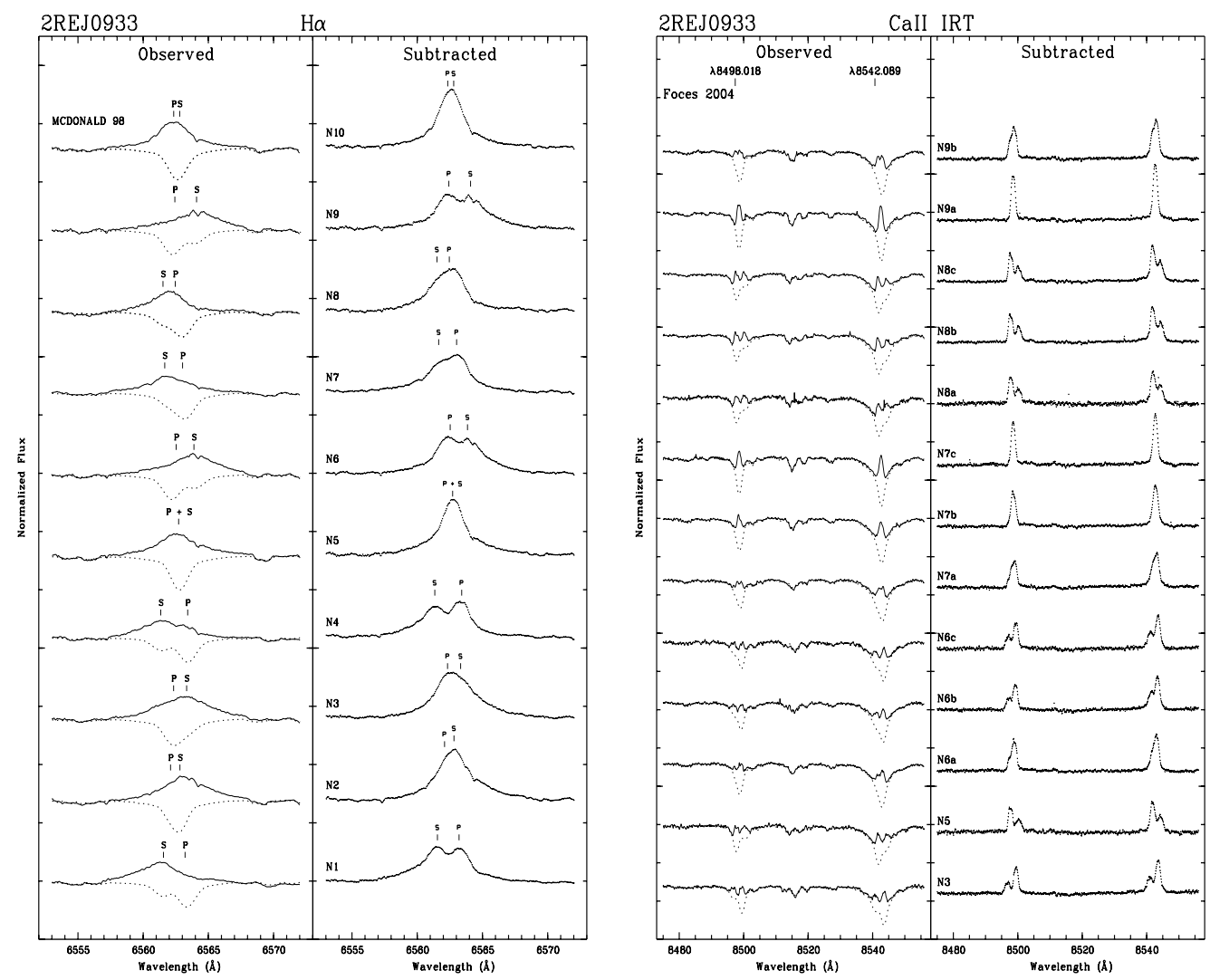

Figure 5. Spectra in $\mathrm{H} \alpha$ line region (left figure) and Ca II IRT region (right figure) in FOCES04 observing run. The observed spectrum (solid-line) and the synthesized spectrum (dashed-line) are plotted in the left panel, and the subtracted spectrum (dotted line), in the right panel.

the hotter star behind, in order to adopt the same criteria used in previous papers. We have obtained a circular orbit with an orbital period of about 3.274 days. As $P_{\text {phot }} \approx$ 3.270 days we can say that it is a synchronous system. The mass ratio is about 2.12 , indicating that the components are very different. The values of all parameters are in agreement with those of Jeffries et al. (1995).

\section{Chromospheric Activity}

The chromospheric contribution in the different optical chromospheric activity indicators has been determined using the spectral subtraction technique Montes et al. (1995, 1997, 1998). The synthesized spectrum was constructed using the program STARMOD developed at Penn State (Barden 1985). We have deblended the emission from both components using a two-Gaussian fit except for the $\mathrm{H} \alpha$ line. The profiles of the $\mathrm{H} \alpha$ and Ca II IRT $(\lambda 8498, \lambda 8542)$ lines are plotted in Figure 5 . For each observation we have plotted the observed spectrum (solid-line) and the synthesized spectrum (dashed-line) in the left panel and the subtracted spectrum (dotted line) in the right panel. The $\mathrm{H} \alpha$ line of both components is always observed in emission above the continuum in the observed 


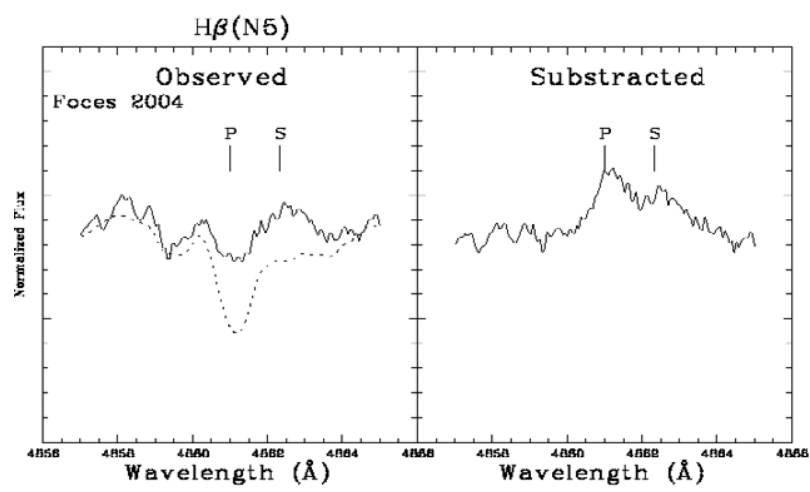

Figure 6. Observed spectrum (solid-line) and the synthesized spectrum (dashed-line) in the left panel, and the subtracted spectrum, in the right panel, in $\mathrm{H} \beta$ line region in FOCES04 observing run.

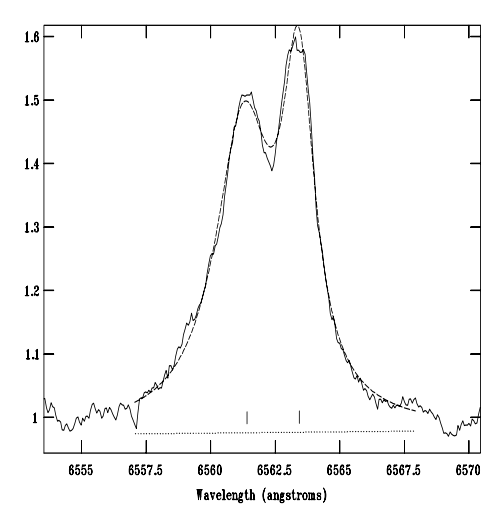

Figure 7. An example of the $\mathrm{H} \alpha$ region fit in the substracted spectrum by two Lorentzians.

spectra (see Figure 5a). This emission is persistent during all the observations, indicating that it is a very active binary system similar to some RS CVn and BY Dra systems.

Measuring the EW of this line, we found that each star of the system has a broad component that does not allow us to fit the emission lines with only one Gaussian. We could not fit the emission lines with four Gaussian components (narrow and broad components for each star). The best fit is obtained when we use a Lorentzian profile for each component (see Figure 7). The other three Balmer lines included in our spectra $(\mathrm{H} \beta, \mathrm{H} \gamma$ and $\mathrm{H} \delta$ ) show a filled-in absorption line profile (see as an example the $\mathrm{H} \beta$ line in Figure 6). A strong emission in the Ca II H\&K lines and a clear emission in the $\mathrm{H} \epsilon$ line coming from both components is also detected. In addition, a clear emission above the continuum is observed in the core of the Ca II IRT absorption lines from both components (see Figure 5b).

\section{Acknowledgements}

This work was supported by the Universidad Complutense de Madrid, the Spanish Ministerio de Educación y Ciencia (MEC), Programa Nacional de Astronomía y Astrofísica under grant AYA2005-02750, and the "Comunidad de Madrid" under PRICIT project S-0505/ESP-0237 (ASTROCAM). 


\section{References}

Applegate J.H. 1992, ApJ 365, 621

Barden S.C. 1985, ApJ 295, 162

Cumming A. 2004, MNRAS 354, 1165

Frasca A. \& Lanza A.F. 2005, ApJ 429, 309

Jeffries R.D., Bertram D. \& Spurgeon R.D. 1995, MNRAS 276, 397

Lanza A.F., Rodono M. \& Rosner R. 1998, MNRAS 296, 893

Lanza A.F. 2006, MNRAS 369, 1773

Matese J.J. \& Whirtmere D.P. 1983, A\&A 117, L7

Montes D., Fernández-Figueroa M.J., De Castro E. \& Cornide M. 1995, A\& A 294, 165

Montes D., Fernández-Figueroa M.J., De Castro E. \& Sanz-Forcada J. 1997, A $\& A$ 125, 263

Montes D., Sanz-Forcada J., Fernández-Figueroa M.J., De Castro E. \& Poncet A. 1998, A\&\&A 330, 155 